\title{
Ultra-wide-field imaging assessment of small choroidal pigmented lesions using red and green colour channels
}

\author{
Vasilios P. Papastefanou (10 ${ }^{1} \cdot$ Rana'a T. Al-Jamal ${ }^{1} \cdot{\text { Zaria Christine } \text { Ali }^{2} \text { • Victoria M. L. Cohen }}^{1} \cdot$ Jane Gray $^{3}$. \\ Mandeep S. Sagoo ${ }^{1,3} \cdot$ Konstantinos Balaskas ${ }^{1,2,4}$
}

Received: 25 February 2019 / Revised: 10 January 2020 / Accepted: 15 April 2020 / Published online: 4 May 2020

(c) The Author(s), under exclusive licence to The Royal College of Ophthalmologists 2020

\begin{abstract}
Background Diagnosis of small choroidal melanoma is mainly based on tumour thickness, subretinal fluid, or lipofuscin pigment. Ultra-wide-field imaging (UWF) allows depiction of choroidal lesions through a red (RC) and a green channel (GC). Aim of the study was to determine the utility of this tool in the detection of small choroidal melanoma.

Methods Retrospective cross-sectional study of patients with small choroidal pigmented lesions up to $3 \mathrm{~mm}$ in thickness. All patients underwent clinical and imaging assessment including UWF. Lesions were subcategorized based on thickness and lesion type. A qualitative assessment ensued using the red and green channels feature.

Results A total of 152 patients were included. Melanotic naevi (76/152,50\%) and small choroidal melanomas $(55 / 152,36 \%)$ were the predominant types. Thickness was $<1 \mathrm{~mm}$ in $30 \%$ (46/152), $1-2 \mathrm{~mm}$ in $46 \%$ (70/152) and $2-3 \mathrm{~mm}$ in $24 \%(36 / 152)$ of cases. Two distinct imaging patterns were noted: dark on RC/undetectable on GC and dark on RC/light on GC. In melanotic naevi the dark on RC/light on GC pattern was significantly associated with increased tumour thickness $(p=0.006)$ and the presence of lipofuscin $(p<0.001)$ suggesting a potential prognostic significance. In small melanomas such an association was not established. The majority of small melanomas manifested a dark on RC/undetectable on GC pattern despite the presence of subretinal fluid and lipofuscin.

Conclusions UWF imaging of choroidal pigmented tumours with red-green channels revealed two distinct patterns. The dark on RC/light on GC pattern was more common in suspicious melanotic naevi, but not in small melanomas. The use of red-green channels is not a reliable diagnostic tool in the early detection of small melanomas.
\end{abstract}

Poster presentation in ARVO 2017; poster presentation in the Royal Congress of Ophthalmologists in Liverpool 2018; oral presentation in the European Ocular Oncology Group Meeting in Siena, Italy 2018.

Supplementary information The online version of this article (https:// doi.org/10.1038/s41433-020-0900-6) contains supplementary material, which is available to authorized users.

Konstantinos Balaskas

konstantinos.balaskas@moorfields.nhs.uk

1 Medical Retina Service/Ocular Oncology Service, Moorfields Eye Hospital, 162 City Road, London EC1V 2PD England, UK

2 Medical Retina Department, Manchester Royal Eye Hospital, Oxford Road, Manchester M13 9WL England, UK

3 Institute of Ophthalmology, University College London, 11-43 Bath Street, London EC1V 9EL England, UK

4 Diagnostics Ophthalmic Angiography and Imaging Services, Manchester Royal Eye Hospital, Oxford Road, Manchester M13 9WL England, UK

\section{Introduction}

Choroidal naevi are common benign tumours found in $6-7 \%$ of the Caucasian population [1,2]. Naevi are often monitored due to the small risk of malignant transformation into life threatening choroidal melanomas [1-6]. Clinical risk factors for malignant transformation of choroidal naevi are well established and ascribe the level of suspicion and hence determine the frequency of monitoring [7].

1Identifying naevi with suspicious features has evolved from colour fundus photography and ultrasound B mode scan, to using optical coherence tomography (OCT) to detect subclinical subretinal fluid and/or autofluorescence to confirm lipofuscin [7]. The Optomap (Optos plc, Dunfermline, Scotland) is a digital ultra-wide-field (UWF) retinal imaging system (Scanning Laser Ophthalmoscope), which provides a non-mydriatic, $82 \%$ view $\left(200^{\circ}\right)$ of the fundus. A single fundus image is captured with two low-powered laser wavelengths: red channel (RC) at $633 \mathrm{~nm}$ and green channel 
Table 1 Ultra-wide-field imaging assessment of small choroidal pigmented lesions using red and green colour channels: demographic and tumour features.

Median Age: 66.0 (mean $64.5 \pm 2.2$, range 26-90 years)

Sex

Female: 81/152 (53\%)

Age: $64.0 \pm 3.2$

Male:71/152 (47\%)

Age: $66.0 \pm 3.0$

Lesion type

Melanotic naevi $(76 / 152,50 \%)$

Amelanotic naevi $(3 / 152,2 \%)$

Halo naevi $(3 / 152,2 \%)$

Suspicious naevi $(15 / 152,10 \%)$

Small choroidal melanomas $(55 / 152,36 \%)$

Lesion thickness

0-1 mm: 46/152, 30\%

1-2 mm: 70/152, 46\%

2-3 mm: 36/152, 24\%

Drusen present: 60/152, $40 \%$

Orange pigment present: $62 / 152,41 \%$

Subretinal fluid present: 40/152, 26\%

Fibrosis present: 52/152, 34\%

(GC) at $532 \mathrm{~nm}$ that scan the retina simultaneously. The single image can be deconstructed to see the individual wavelength views. The GC permits a view from the sensory retina to the retinal pigment epithelium (RPE), and the RC scans the deeper structures from the RPE to the choroid.

The aim of this study is to examine the potential use of the red-green channel feature of UWF imaging in the early detection of a suspicious choroidal naevus or a small choroidal melanoma through the assessment of a large series of small choroidal pigmented lesions.

\section{Methods}

This is a retrospective study of patients referred for further assessment of pigmented fundus lesions to Manchester Royal Eye Hospital and Moorfields Eye Hospital from January 2016 to January 2018. As part of their routine care, these patients underwent a series of imaging tests performed by trained imaging technicians including ultrasound B-scan, autofluorescence, OCT and UWF imaging (Optos California and 200Tx-Optos plc, Dunfermline, Scotland)

Patient demographics and tumour features (thickness, drusen, orange pigment, subretinal fluid and fibrosis) were recorded. The UWF images were then reviewed in both the red and green channels by adjusting the blend function of the software.
Lesions were subcategorized based on thickness on Bscan ultrasound $(0-1,1-2$ and $2-3 \mathrm{~mm})$ and according to lesion type (melanotic choroidal naevi, amelanotic choroidal naevi, halo naevi, suspicious naevi and small melanomas). Detectability was qualitatively characterized as Dark, Light or not detectable following assessment by two experienced ophthalmologists (VP, CAZ).

Descriptive statistical analysis with the $x^{2}$ and $t$-test were used to evaluate the findings after prior Kolmogorov-Smirnov testing for normal distribution of the results, A difference of 0.05 was considered statistically significant. Analysis was done with Wizard Pro 1.9 .26 (USA)

\section{Results}

\section{Patient demographics and tumours}

A total of 152 cases were included in this study. Patient demographics and tumour features are summarized in Table 1 . The lesions assessed were melanotic naevi (76/152, $50 \%)$, amelanotic naevi $(3 / 152,2 \%)$, halo naevi $(3 / 152$, $2 \%)$, suspicious naevi $(15 / 152,10 \%)$ and small choroidal melanomas $(55 / 152,36 \%)$. The two predominant categories, namely, melanotic naevi and small melanomas were analyzed. All choroidal melanomas were stage I (T10 N0 M0) based on the 8th Edition of AJCC classification [8].

\section{Red vs green channel analysis on the two predominant tumour types}

\section{Melanotic naevi}

There were 76 cases in the melanotic naevus group. The mean tumour thickness on ultrasound was $0.9 \pm 0.1 \mathrm{~mm}$. The clinical features of the melanotic naevus group are presented in Table 2A. RC and GC yielded two principal patterns of detectability: (a) Dark on RC/undetectable on GC (57/76, 75\%); and (b) Dark on RC/light on GC (15/76, 20\%) (Table 2A, Figs. 1 and 2).

Analysis of the clinical features of the lesions with respect to these two patterns showed some differences. The dark on RC/light on GC pattern occurred in slightly thicker tumours $(1.2 \pm 0.2 \mathrm{~mm}$ vs $0.8 \pm 0.2 \mathrm{~mm}, p=0.006)$ and in the presence of orange pigment $(27 \%$ vs $2 \%, p<0.001)$. The presence of subretinal fluid ( $7 \%$ vs $2 \%, p=0.3)$ did not reach statistical significance (Table $2 \mathrm{~A}$ ).

\section{Small choroidal melanomas}

The small melanoma group comprised 55 cases. The mean tumour thickness was $2.7 \pm 0.3 \mathrm{~mm}$. The clinical features of 
Table 2 Ultra-wide-field imaging assessment of small choroidal pigmented lesions using red and green colour channels.

\begin{tabular}{|c|c|c|c|c|c|c|c|c|}
\hline \multicolumn{9}{|c|}{ A Features of melanotic naevus group } \\
\hline & \multicolumn{3}{|c|}{ All melanotic naevi } & \multicolumn{2}{|l|}{$\begin{array}{l}\text { Dark on RC } \\
\text { undetectable in GC }\end{array}$} & \multicolumn{2}{|l|}{$\begin{array}{l}\text { Dark on RC } \\
\text { Light on GC }\end{array}$} & \multirow[t]{2}{*}{$p$ value* (test) } \\
\hline & & $\begin{array}{l}\text { Median }(\text { Mean } \pm \text { SD }) \\
\text { Or } n / N(\%)\end{array}$ & Range & $\begin{array}{l}\text { Median (Mean } \pm \text { SD) } \\
\text { Or } n / N(\%)\end{array}$ & Range & $\begin{array}{l}\text { Median }(\text { Mean } \pm \text { SD) } \\
\text { Or } n / N(\%)\end{array}$ & Range & \\
\hline No of patients & & 76 & & $57 / 76(75 \%)$ & & $15 / 76(20 \%)^{\mathrm{a}}$ & & \\
\hline $\begin{array}{l}\text { Ultrasound } \\
\text { thickness (mm) }\end{array}$ & & $0.9(0.92 \pm 0.1)$ & $0.25-2.2$ & $0.8 \pm 0.2$ & $0.1-2.2$ & $1.2 \pm 0.2$ & $0.6-2$ & $0.006(t$-test $)$ \\
\hline \multirow{3}{*}{$\begin{array}{l}\text { Ultrasound thickness } \\
\text { distribution }(\mathrm{mm})\end{array}$} & $0-1$ & $64 / 76(85 \%)$ & & $36 / 57(63 \%)$ & & $3 / 15(20 \%)$ & & \\
\hline & $1-2$ & $39 / 76(51 \%)$ & & $20 / 57(35 \%)$ & & $12 / 15(80 \%)$ & & \\
\hline & $2-3$ & $2 / 76(3 \%)$ & & $1 / 57(2 \%)$ & & $0 / 15(0 \%)$ & & \\
\hline \multicolumn{9}{|l|}{ Clinical features } \\
\hline Drusen & & $33 / 76(43 \%)$ & & $25 / 57(44 \%)$ & & $5 / 15(33 \%)$ & & $0.2\left(x^{2}\right)$ \\
\hline Orange pigment & & $6 / 76(8 \%)$ & & $1 / 57(2 \%)$ & & $4 / 15(27 \%)$ & & $<0.001\left(x^{2}\right)$ \\
\hline Subretinal fluid & & $2 / 76(3 \%)$ & & $1 / 57(2 \%)$ & & $1 / 15(7 \%)$ & & $0.303\left(x^{2}\right)$ \\
\hline Fibrosis & & $14 / 76(19 \%)$ & & $7 / 57(12 \%)$ & & $5 / 15(33 \%)$ & & $0.052\left(x^{2}\right)$ \\
\hline \multicolumn{9}{|c|}{ B Small choroidal melanoma group } \\
\hline & \multicolumn{2}{|c|}{ All small melanomas } & & $\begin{array}{l}\text { Dark on RC } \\
\text { undetectable on GC }\end{array}$ & & $\begin{array}{l}\text { Dark on RC } \\
\text { Light on GC }\end{array}$ & & $p$ value* (test) \\
\hline & & $\begin{array}{l}\text { Median }(\text { Mean } \pm \text { SD }) \\
\text { Or } n / N(\%)\end{array}$ & Range & $\begin{array}{l}\text { Median }(\text { Mean } \pm \text { SD }) \\
\text { Or } n / N(\%)\end{array}$ & Range & $\begin{array}{l}\text { Median }(\text { Mean } \pm \text { SD) } \\
\text { Or } n / N(\%)\end{array}$ & Range & \\
\hline No of patients & & $55 / 152(37 \%)$ & & $40 / 55(73 \%)$ & & $12 / 55(22 \%)^{\mathrm{b}}$ & & \\
\hline Thickness (mm) & & $2.1(2.2 \pm 0.1)$ & $1-3.0$ & $2 \pm 0.2$ & & $2.7 \pm 0.3$ & & $0.006(t$-test $)$ \\
\hline \multirow[t]{3}{*}{ Thickness distribution } & $0-1$ & $1 / 55(1.8 \%)$ & & $1 / 40(2.5 \%)$ & & $0 / 12(0 \%)$ & & \\
\hline & $1-2$ & $24 / 55(43.6 \%)$ & & $21 / 40(52.5 \%)$ & & $1 / 12(8 \%)$ & & \\
\hline & $2-3$ & $30 / 55(54.5 \%)$ & & $18 / 40(45 \%)$ & & $11 / 12(92 \%)$ & & \\
\hline Drusen & & $19 / 55(34.55 \%)$ & & $12 / 40(30 \%)$ & & $6 / 12(50 \%)$ & & $0.2\left(x^{2}\right)$ \\
\hline Orange pigment & & $47 / 55(85.4 \%)$ & & $37 / 40(92.5 \%)$ & & $8 / 12(26.7 \%)$ & & $<0.001\left(x^{2}\right)$ \\
\hline Subretinal fluid & & $30 / 55(54.5 \%)$ & & $24 / 40(60 \%)$ & & $6 / 12(50 \%)$ & & $0.3\left(x^{2}\right)$ \\
\hline Fibrosis & & $32 / 55(58 \%)$ & & $24 / 40(60 \%)$ & & $6 / 12(50 \%)$ & & $0.052\left(x^{2}\right)$ \\
\hline
\end{tabular}

$G C$ green channel; $R C$ red channel.

$* p<0.05$ considered statistically significant, $t$-test or $\mathrm{x}^{2}$.

${ }^{a}$ Five cases did not fit in any of the patterns and are not included in the table.

${ }^{\mathrm{b}}$ Three cases did not fit in any of the patterns and are not included in the table.

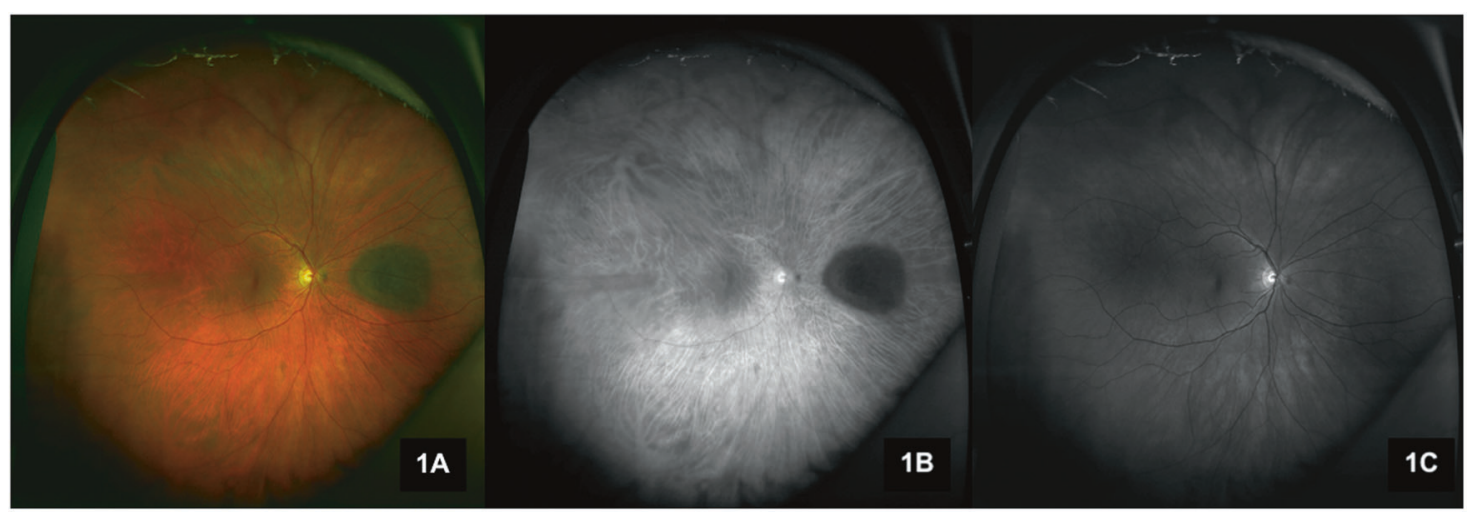

Fig. 1 Optos photo of a flat naevus. a Colour photo. b Naevus appears dark in red channel. c Naevus is not visible in green channel (dark on red - not detectable on green pattern). 


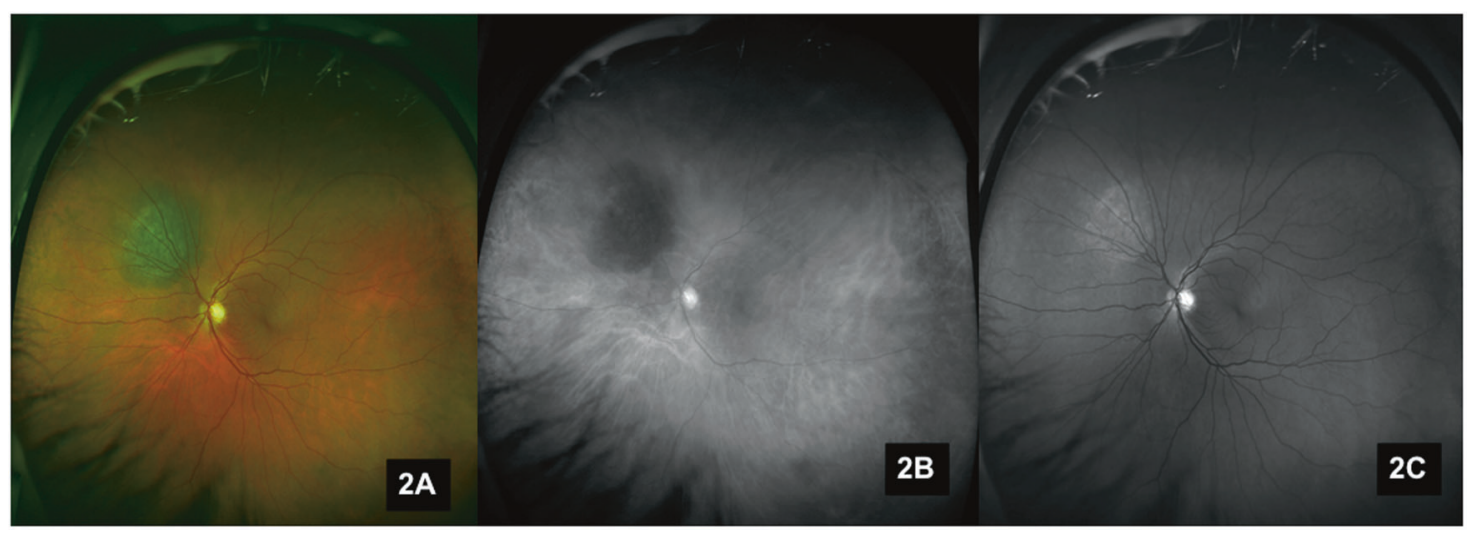

Fig. 2 Optos photos of an elevated naevus (1.2 mm). a Colour photo. b Naevus appear dark in red channel. c Naevus appears bright in green channel (Dark on red-light on green pattern).

Table 3 Ultra-wide-field imaging assessment of small choroidal pigmented lesions using red and green colour channels.

\begin{tabular}{|c|c|c|c|c|c|c|}
\hline \multicolumn{7}{|c|}{ A Clinical features by tumour thickness category } \\
\hline Tumour thickness category & $0-1 \mathrm{~mm}$ & $1-2 \mathrm{~mm}$ & \multicolumn{4}{|l|}{$2-3 \mathrm{~mm}$} \\
\hline No of patients & 46 & 70 & \multicolumn{4}{|l|}{36} \\
\hline $\begin{array}{l}\text { Thickness }(\mathrm{mm}) \text { median } \\
(\text { mean } \pm \mathrm{SD})\end{array}$ & $0.5(0.5 \pm 0.06)$ & $1.2(1.4 \pm 0.07)$ & \multicolumn{4}{|l|}{$2.5(2.6 \pm 0.1)$} \\
\hline \multicolumn{7}{|c|}{ Distribution of tumour type per thickness category } \\
\hline Melanotic naevus (\%) & 85 & 51 & \multicolumn{4}{|l|}{2.8} \\
\hline Amelanotic naevus (\%) & 7 & & & & & \\
\hline Halo naevus $(\%)$ & 0 & 0 & \multicolumn{4}{|l|}{0} \\
\hline $\begin{array}{l}\text { Indeterminate melanocytic } \\
\text { lesion }(\%)\end{array}$ & 4.3 & 11.4 & \multicolumn{4}{|l|}{13.9} \\
\hline $\begin{array}{l}\text { Small choroidal } \\
\text { melanoma }(\%)\end{array}$ & 2.2 & 34.3 & \multicolumn{4}{|l|}{83.3} \\
\hline \multicolumn{7}{|l|}{ Tumour features } \\
\hline Drusen present $(\%)$ & 41.3 & 37.1 & \multicolumn{4}{|l|}{41.7} \\
\hline Orange pigment present $(\%)$ & 6.5 & 44.3 & \multicolumn{4}{|l|}{77.8} \\
\hline Subretinal fluid present (\%) & 6.5 & 21.4 & \multicolumn{4}{|l|}{61.1} \\
\hline Fibrosis present $(\%)$ & 8.7 & 34.3 & \multicolumn{4}{|l|}{66.7} \\
\hline \multicolumn{7}{|c|}{ B Detectability of clinical features by red and green channel } \\
\hline \multirow[t]{2}{*}{ Tumour type } & Drusen & & \multicolumn{2}{|c|}{ Orange pigment } & \multicolumn{2}{|c|}{ Subretinal fluid } \\
\hline & $\begin{array}{l}\text { Red } \\
\text { channel }\end{array}$ & $\begin{array}{l}\text { Green } \\
\text { channel }\end{array}$ & Red channel & $\begin{array}{l}\text { Green } \\
\text { channel }\end{array}$ & $\begin{array}{l}\text { Red } \\
\text { channel }\end{array}$ & $\begin{array}{l}\text { Green } \\
\text { channel }\end{array}$ \\
\hline Melanotic naevus & $26 / 76$ & $25 / 76$ & $1 / 76$ & $1 / 76$ & & $1 / 76$ \\
\hline CMM & $16 / 55$ & $18 / 55$ & $42 / 55$ & $40 / 55$ & $11 / 55$ & $20 / 55$ \\
\hline IML & $4 / 15$ & $5 / 15$ & $6 / 15$ & $4 / 15$ & $1 / 15$ & $6 / 15$ \\
\hline Halo naevus & $2 / 3$ & $2 / 3$ & $1 / 3$ & & & \\
\hline Amelanotic naevus & 0 & 0 & & & & \\
\hline
\end{tabular}

the small melanoma group are presented in Table $2 \mathrm{~B}$. RC and GC gave the same two patterns of detectability: (a) Dark on RC/undetectable on GC (40/55, 73\%) and (b) dark on RC/light on GC (12/55, 22\%).

Analysis of the clinical features of small melanomas with respect to these two patterns showed similar differences. The dark on RC/light on GC pattern was associated with increased tumour thickness $(2.7 \pm 0.3 \mathrm{~mm}$ vs $2 \pm 0.2 \mathrm{~mm}, p$
$<0.001$ ), a finding that occurred even in the $2-3 \mathrm{~mm}$ in tumour thickness subgroup. Despite this trend with tumour size, the majority of small melanomas displayed the other pattern, dark on RC/undetectable on GC (40/55, 73\%). Contrary to the naevus cohort, the presence of orange pigment was associated more commonly in melanomas with the dark on RC/undetectable on GC pattern $(93 \%$ vs $67 \%, p=0.02$ ). 


\section{$\mathrm{RC} / \mathrm{GC}$ analysis based on tumour thickness}

All lesions were categorized by tumour thickness into three groups, namely $0-1 \mathrm{~mm}(n=46$, median $0.5 \mathrm{~mm}$, mean $0.5 \pm$ $0.06), 1-2 \mathrm{~mm}(n=70$, median 1.2 , mean $1.4 \pm 0.07)$ and $2-3 \mathrm{~mm}(n=36$, median 2.5 , mean $2.6 \pm 0.1)$. The features of each group are presented in Table 3. The proportion of dark on RC/light on GC pattern increased according to the thickness of the tumour, $8.9 \%$ in the $0-1 \mathrm{~mm}$ thickness group, $24.6 \%$ in the $1-2 \mathrm{~mm}$ group and $30.6 \%$ in the $2-3 \mathrm{~mm}$ group $(p=0.04)$ (Supplementary Fig. 3A).

We also analyzed the RC/GC pattern to determine the association with size (Supplementary Fig. 3B). In the dark on $\mathrm{RC} /$ light on GC group, $13 \%$ of lesions were $0-1 \mathrm{~mm}$, $52 \%$ were $1-2 \mathrm{~mm}$ and $36 \%$ were $2-3 \mathrm{~mm}$. In the dark on $\mathrm{RC} /$ undetectable on GC group $36 \%$ were $0-1 \mathrm{~mm}, 43 \%$ were $1-2 \mathrm{~mm}$ and $22 \%$ were $2-3 \mathrm{~mm}$ thickness. By thickness, the two patterns differed with statistical significance only in the $0-1 \mathrm{~mm}$ group $\left(x^{2}=0.015\right)$.

\section{Red vs green channel analysis based on tumour features}

\section{Drusen}

Drusen were present in 60/152 (39\%) tumours. The distribution of drusen was equal for all thickness categories (Table 3A) and equivalent between the two channels (Table 3B). There was no correlation with the lesion detectability patterns $\left(x^{2}=0.65\right)$ (Supplementary Fig. 4A)

\section{Orange pigment}

Orange pigment was present in 62/152 (41\%) of tumours. The frequency of orange pigment increased by thickness categories-7\% in $0-1 \mathrm{~mm}$ tumours, $44 \%$ in $1-2 \mathrm{~mm}$ tumours and $78 \%$ in $2-3 \mathrm{~mm}$ tumours, respectively (Table 3A). Orange pigment detection was equivalently distributed between the two channels (Table 3B) but did not correlate with lesion detectability patterns $\left(x^{2}=0.66\right)$ (Supplementary Fig. 4B)

\section{Subretinal fluid}

Subretinal fluid was present in 40/152 (26\%) tumours. The frequency of subretinal fluid increased with thickness categories $-7 \%$ in $0-1 \mathrm{~mm}$ tumours, $21 \%$ in $1-2 \mathrm{~mm}$ tumours and $61 \%$ in $2-3 \mathrm{~mm}$ tumours, respectively (Table 3A). Subretinal fluid was better detected in the GC for 20/55 small CMM and 6/15 IML (Table 3B). The lesion detectability patterns did not correlate with the presence of subretinal fluid $\left(x^{2}=0.8\right)$ (Supplementary Fig. 4C)

\section{Fibrosis}

Overlying fibrosis was present in 52/152 (34\%) tumours. Similarly, there was an increase in frequency of fibrosis by thickness groups-9\% in $0-1 \mathrm{~mm}$ tumours, $34 \%$ in $1-2 \mathrm{~mm}$ tumours and $67 \%$ in $2-3 \mathrm{~mm}$ tumours, respectively (Table 3A). The distribution was identical between channels (Table 3B). The lesion detectability patterns did not correlate with the presence of fibrosis $\left(x^{2}=0.3\right)$ (Supplementary Fig. 4D).

\section{Discussion}

Choroidal naevi are common benign intraocular tumours with prevalence in large population studies of $\sim 7 \%$, though it may be as high as $20-25 \%$ [1]. It is therefore important to detect suspicious features at an early stage [1-6]. Melanocytic fundus tumours that have one suspicious feature have a $38 \%$ chance for growth at five years, rising to $50 \%$ for those with two or more suspicious features [7].

In recent years, modern imaging modalities have been harnessed in the assessment of choroidal lesions. OCT has improved visibility of the choroid as enhanced depth imaging or swept-source OCT [9, 10], autofluorescence scans have confirmed lipofuscin deposition [11] and OCT angiography may give information on blood flow [12].

UWF imaging has been an important addition in this array of imaging modalities because of the ability of capture a wide area of the fundus. In the assessment of intraocular tumours, it has allowed better depiction of the extent of sizable or very peripheral tumours not easily captured with previously available cameras [13]. In light of this principal advantage UWF imaging has also been adopted in the community in optometry practices as part of a quick means to assess the fundus and help in the identification of peripherally located disease.

This study examined this feature of the dual laser imaging platform. The blend function allows separation of the fundus through two distinct channels, the green laser (GC) scanning from the sensory retina to the RPE, and the red laser (RC) scanning the deeper structures from the RPE to the choroid. A large cohort of patients with pigmented choroidal lesions was assessed and different categories were included with the predominant ones being benign melanotic choroidal naevi and small choroidal melanomas. The aim of this study was to determine whether RC and GC separation would allow the identification early signs of transformation from naevus to melanoma. Previous studies [14] suggested that malignant melanomas appeared dark in the RC and bright in the GC with the use of this imaging platform. In addition, we subcategorized lesions by thickness in order to identify any distinctive features for growth, including the 
2-3 mm size of small melanomas. This is the threshold for small choroidal melanoma based on the 8th edition AJCC classification [8]. Detection of small melanomas is important as early treatment may impact on mortality [7].

Our RC and GC patterns analysis compared benign melanotic naevi and small choroidal melanomas. Indeterminate melanocytic lesions and suspicious naevi were excluded due to their small numbers in this cohort. The important finding in this first comparison was the presence of two distinct patterns on RC and GC in the vast majority of cases namely that $72 / 76$ cases in the melanotic naevi group and 52/55 cases in the small choroidal melanoma group were either dark on RC/undetectable on GC or dark on RC /light on GC. The remaining seven cases were not clearly characterized and therefore not included in this analysis.

Analysis of the predominant patterns indicated that dark on RC/light on GC melanotic naevi were significantly thicker, significantly associated with the presence of orange pigment and there was a trend towards subretinal fluid. In the case of established small melanomas however, the vast majority were dark on RC/undetectable on GC.

Kernt et al. [14] found that dark on RC/light on GC was distinctive of malignant melanoma. Their study compared 29 choroidal melanomas with 20 naevi but there are some differences to our study. Our lesions were smaller compared to sizes ranging from 7 to $20 \mathrm{~mm}$ therefore including stages II-IV lesions [15]. Apart from size the only other clinical feature evaluated by Kernt et al. [14] was the presence or absence of subretinal fluid, whereas we examined the presence of drusen, orange pigment, subretinal fluid and fibrosis and whether these affect the detectability of the lesion. These differences may affect the disparity of our results. In the choroidal naevi group the presence of the dark on RC/light on GC pattern indeed increases the index of suspicion for the fraction of tumours falling under this category. These were lesions with greater thickness and higher chance of lipofuscin and subretinal fluid.

Extrapolating from this result in the naevus group, the small melanoma group should also manifest dark on $\mathrm{RC} /$ light on GC pattern. This was not the case. Despite a significantly greater tumour thickness, the vast majority of small choroidal melanomas were not detectable with GC and importantly the lipofuscin deposits spotted clinically were not detectable either at a significantly higher rate. Autofluorescence scans remain important in the assessment of lipofuscin deposits [9]. On the other hand the RC may be superior to autofluorescence for the follow up of choroidal naevi, due to deeper penetration [15].

When this cohort was analyzed with tumour thickness as the sole parameter the dark on RC/light on GC pattern tended to increased significantly from $8.9 \%$ in the $0-1 \mathrm{~mm}$ category to $30.6 \%$ in the $2-3 \mathrm{~mm}$ category (Supplementary
Fig. 3A). However among dark to light lesions falling under the $1-2 \mathrm{~mm}$ and $2-3 \mathrm{~mm}$ categories frequency of dark on $\mathrm{RC} /$ light on GC was higher but not significant (Supplementary Fig. 3B).

Would therefore the dark on RC/light on GC pattern be of prognostic significance? As the GC penetrates through the neurosensory retina up to the RPE layer, it is difficult to claim that it could capture an inherent property of the underlying choroidal lesion, such as a metabolic or vascular feature indicative of growth. A plausible explanation is that the lighter signal corresponds to RPE dysfunction a common event in both chronic naevi of a certain size and in small choroidal melanomas. This event in itself however does not confer prognostic significance [7].

Examination of individual clinical features showed that drusen, orange pigment and fibrosis were equally distributed between channels. These clinical features do not correlate significantly with a particular RC or GC pattern and therefore one cannot predict their presence from the channel patterns.

In conclusion, despite the promising notion that the use of two separate channels might detect a malignant lesion early and add to its prognosis, our results do not support this. Melanotic naevi and small melanomas appear dark on RC. Benign or malignant melanocytic tumours can appear light on GC possibly due to the chronicity of the lesion but not due to an inherent property related to prognostic significance. $\mathrm{RC}$ has the benefit of assess tumour margins in serial monitoring, but RC and GC patterns are limited in differentiating choroidal naevi from melanomas.

\section{Summary}

\section{What was known before}

- Choroidal pigmented lesions are clinically assessed based on known risk factors red-green channel feature of ultra-wide-field imaging is a useful clinical tool.

- This feature has been previously suggested to discern choroidal naevi from melanomas.

\section{What this study adds}

- Red-green channel features allow the detection of specific patterns in pigmented lesions.

- These patterns are not distinctive in early differential diagnosis between choroidal naevi and small melanomas.

- Red-green channels do not significantly correlate with known suspicious clinical features of choroidal pigmented lesions as lipofuscin or subretinal fluid. 


\section{Compliance with ethical standards}

Conflict of interest The authors declare that they have no conflict of interest.

Publisher's note Springer Nature remains neutral with regard to jurisdictional claims in published maps and institutional affiliations.

\section{References}

1. Chien JL, Sioufi K, Surakiatchanukul T, Shields JA, Shields CL. Choroidal nevus: a review of prevalence, features, genetics, risks, and outcomes. Curr Opin Ophthalmol. 2017;28:228-37.

2. Shields CL, Furuta M, Berman EL, Zahler JD, Hoberman DM, Dinh DH, et al. Choroidal nevus transformation into melanoma: analysis of 2514 consecutive cases. Arch Ophthalmol. 2009;127:981-7.

3. Mims JL, 3rd, Shields JA. Follow-up studies of suspicious Choroidal Nevi. Ophthalmology. 1978;85:929-43. https://doi.org/ 10.1016/s0161-6420(78)35597-4

4. Gass JDM. Observation of suspected choroidal and ciliary body melanomas for evidence of growth prior to enucleation. Ophthalmology. 87:523-28. https://doi.org/10.1016/S0161-6420(80) 35200-7

5. Butler P, Char DH, Zarbin M, Kroll S. Natural history of indeterminate pigmented choroidal tumors. Ophthalmology. 101:710-17

6. Augsburger JJ, Schroeder RP, Territo C, Gamel JW, Shields JA. Clinical parameters predictive of enlargement of melanocytic choroidal lesions. Br J Ophthalmol. 1989;73:911-7.
7. Shields CL, Shields JA. Clinical features of small choroidal melanoma. Curr Opin Ophthalmol. 2002;13:135-41.

8. Amin MB, Edge SB, Greene FL, Byrd DR, eds. AJCC Cancer Staging Manual. 8th ed New York, NY: Springer. 2017;805-17

9. Francis JH, Pang CE, Abramson DH, Milman T, Folberg R, Mrejen S, et al. Swept-source optical coherence tomography features of choroidal nevi. Am J Ophthalmol. 2015;159:169-76.

10. Shields CL, Kaliki S, Rojanaporn D, Ferenczy SR, Shields JA. Enhanced depth imaging optical coherence tomography of small choroidal melanoma: comparison with choroidal nevus. Arch Ophthalmol. 2012;130:850-6.

11. Albertus DL, Schachar IH, Zahid S, Elner VM, Demirci H, Jayasundera $\mathrm{T}$. Autofluorescence quantification of benign and malignant choroidal nevomelanocytic tumors. JAMA Ophthalmol. 2013;131:1004-8

12. Ali ZC, Gray J, Balaskas K. Features of choroidal naevi on swept source optical coherence tomography angiography and structural reverse flow optical coherence tomography. Graefes Arch Clin Exp Ophthalmol. 2018;256:1319-23.

13. Coffee RE, Jain A, McCannel TA. Ultra wide-field imaging of choroidal metastasis secondary to primary breast cancer. Semin Ophthalmol. 2009;24:34-6.

14. Kernt M, Schaller UC, Stumpf C, Ulbig MW, Kampik A, Neubauer AS. Choroidal pigmented lesions imaged by ultra-wide-field scanning laser ophthalmoscopy with two laser wavelengths (Optomap). Clin Ophthalmol. 2010;4:829-36.

15. Zapata MA, Leila M, Teixidor T, Garcia-Arumi J. Comparative study between fundus autofluorescence and red reflectance imaging of choroidal nevi using ultra-wide-field scanning laser ophthalmoscopy. Retina. 2015;35:1202-10. 